\title{
VEGF, eNOS, and $A B C B 1$ genetic polymorphisms may increase the risk of osteonecrosis of the femoral head
}

\author{
Z.C. Zhou, S.Z. Gu, J. Wu and Q.W. Liang \\ Department of Orthopedics, \\ The First Affiliated Hospital of China Medical University, \\ Shenyang, Liaoning Province, China \\ Corresponding author: Q.W. Liang \\ E-mail: cmulqw@126.com
}

Genet. Mol. Res. 14 (4): 13688-13698 (2015)

Received May 3, 2015

Accepted July 14, 2015

Published October 28, 2015

DOI http://dx.doi.org/10.4238/2015.October.28.31

\begin{abstract}
We investigated the associations between vascular endothelial growth factors (VEGF), endothelial nitric oxide synthase (eNOS), and ATP-binding cassette subfamily $\mathrm{B}$ member 1 transporter (ABCB1) polymorphisms and the risk of osteonecrosis of the femoral head (ONFH). Published studies were reviewed and analyzed based on predefined selection criteria. The strength of the association between VEGF, eNOS, and $A B C B 1$ polymorphisms and ONFH risk was evaluated based on the odds ratio with corresponding $95 \% \mathrm{Cls}$. Meta-analysis was performed using the Comprehensive Meta-analysis 2.0 software. A total of 135 relevant articles were retrieved, of which 10 studies met the selection criteria, and included a total of 1025 patients with ONFH and 1730 healthy controls. The meta-analysis study results revealed that the VEGF rs2010963 G>C polymorphism increased the risk of ONFH, while the VEGF rs2010963 $\mathrm{G}>\mathrm{C}$ and $A B C B 1$ rs1045642 $\mathrm{C}>\mathrm{T}$ polymorphisms increased the risk of
\end{abstract}


ONFH under the allele model. In conclusion, the VEGF, eNOS, and ABCB1 polymorphisms may contribute to ONFH, but further studies including larger sample sizes are needed to confirm the results.

Key words: ATP-binding cassette subfamily B member 1 transporter; Endothelial nitric oxide synthase; Osteonecrosis of the femoral head; Meta-analysis; Polymorphism; Vascular endothelial growth factor gene

\section{INTRODUCTION}

Rheumatoid arthritis occurs in 23.7 per 100,000 individuals each year, and osteoarthritis of the hip joint results in 47.3-88 cases per 100,000 people every year in the US (Yamaguchi et al., 2011). Osteonecrosis (ON) is a pathological process in which cellular death in the bone constituents occurs because of decreased blood flow or an interruption in the blood supply (Kim et al., 2008). ON of the femoral head (ONFH) is one of the most common diseases of ON, with 10,000-20,000 new cases annually in the US alone; ONFH occurs mainly in young and active individuals between 30 and 50 years old (Gagala et al., 2013). Previous studies linked the pathogenesis of ONFH to an interruption of blood circulation to the anterior superior lateral components of the femoral head, resulting in ischemic insult and bone collapse. ONFH may also arisedue to trauma, alcoholism, glucocorticoid therapy, or storage diseases (Kim et al., 2009; Liang et al., 2013). Recent studies have suggested that an association exists between ONFH and genetic polymorphisms in the vascular endothelial growth factor (VEGF), endothelial nitric oxide synthase (eNOS), and ATP-binding cassette subfamily B member 1 transporter (ABCB1) genes (Koo et al., 2006; Wang et al., 2011; Liu et al., 2012). These studies aimed to identify new targets for ONFH treatment.

$V E G F$, located on chromosome $6 \mathrm{p} 31.3$, is the principal molecule involved in angiogenesis during normal embryogenesis and is closely related to several critical biological processes in adults such as regeneration, neuroprotection, neuronal survival, growth, differentiation, and migration (Cariboni et al., 2011; Foxton et al., 2013; Gao et al., 2013). Polymorphisms located in the 5 '-promoter regionalters VEGF expression and may affect the etiology of numerous pathological conditions including diabetic retinopathy, prostate cancer, breast cancer, and ONFH (Guan et al., 2009; Lee et al., 2012). Nitric oxide (NO) impedes platelet activation, adhesion, and aggregation (Dangel et al., 2010; Herrera et al., 2011). Furthermore, NO is an intracellular messenger that plays a vital role in vascular system, homoeostasis, and bone turnover, all of which are closely related to the ONFH disease process (Koo et al., 2006). The constitutive isoform of NOS, eNOS, is responsible for maintaining the basal level of NO production and is important for bone growth and metabolism (Harvey et al., 2012). Human eNOS is mapped to chromosome 7q35-36, is composed of 26 exons and 25 introns, and encodes a 133-kDa protein product (Mehrab-Mohseni et al., 2011; Higashibata et al., 2012). A few previous studies observed that some polymorphisms in eNOS result in lower NO synthesis because of reductions in eNOS expression; these polymorphisms have been correlated with the risk of ONFH (Kooet al., 2006). P-glycoprotein (P-gp), encoded by $A B C B 1$, has also been correlated to the development of ONFH, and increased P-gp activity reduces steroid-induced ONFH risk (Han et al., 2010). $A B C B 1$ (also known as multidrug resistance protein-1 or MDR1), is an efflux transporter that impacts the interaction profiles and disposal kinetics of several useful pharmacological drugs, such as cyclosporin, digoxin, and fexofenadine (Alzoubi 
et al., 2013; García et al., 2013). The rs1045642 C>T polymorphism located at position 3435 in exon 26 of $A B C B 1$ alters P-gp expression levels and may influence ONFH disease progression (Fungand Gottesman, 2009; Li et al., 2014). However, the roles of VEGF, eNOS, and $A B C B 1$ polymorphisms in ONFH remain unclear and are highly disputed (Kim et al., 2013). Thus, in this meta-analysis, we investigated the risk factors of ONFH disease progression, focusing on selected polymorphisms within these 3 genes.

\section{MATERIAL AND METHODS}

\section{Literature search}

Original published studies were retrieved from electronic databases including PubMed (1966-October, 2014), Cochrane Library (CEN-TRAL, 2014), Ovid (1948-October, 2014), Embase (1966-October, 2014), China National Knowledge Infrastructure (CNKI, 1994-October, 2014), and Wanfang (1986-October, 2014). Manual searches were additionally employed to identify relevant articles from cross-references. The search key words included: "osteonecrosis of the femoral head", "ONFH", "vascular endothelial growth factors", "VEGF", "endothelial nitric oxide synthase", "eNOS", "ABCB1", and "polymorphism".

\section{Selection criteria}

Two investigators independently reviewed the titles and abstracts of all identified literature and selected a list of potential studies for more detailed analysis. The full texts of these studies were reviewed to assess whether the studies were suitable for final inclusion. The studies were enrolled if they met the following inclusion criteria: 1) case-control studies; 2) studies containing correlations between VEGF, eNOS, and $A B C B 1$ polymorphisms and ONFH; 3) the case group included patients with $\mathrm{ONFH}$, while the control group included healthy individuals; 4) studies provided complete information, including year of publication, country of origin, ethnicity, language, age, gender, genotyping method, case group numbers, number of cases, and genotype distributions of VEGF rs2010963 G>C, eNOS rs2070744 T>C, ABCB1 rs1045642 $\mathrm{C}>\mathrm{T}$. Our exclusion criteria were: 1) the diagnostic criteria were undefined; 2) duplicated articles; 3) studies with incomplete data.

\section{Data extraction}

Both investigators extracted data independently from the enrolled studies by manual review. Discrepancies in the data were resolved by discussion with a third investigator. The extracted information included the first author's name, publication year, ethnicity, language, disease, sample size, age, genotyping method, and genotype distributions of VEGF rs2010963 $\mathrm{G}>\mathrm{C}$, eNOS rs2070744 T>C, and ABCB1 rs1045642 C>T. Study quality was determined by the 2 reviewers using the critical appraisal skill program (CASP) (http://www.casp-uk.net/). The CASP criteria are scored based on following aspects: 1) the study addressed a clearly focused issue (CASP01); 2) the authors used an appropriate method to answer their question (CASP02); 3) the cases were recruited in an acceptable manner (CASP03); 5) the controls were selected in an acceptable manner (CASP04); the exposure was accurately measured to minimize bias (CASP05); 
6) the authors have taken account of the potential confounding factors in the design and/or in their analysis (CASP06); 7) what are the results of this study (CASP07); 8) the results and the estimate of risk are precise (CASP08); 9) the results are believable (CASP09); 10) the results can be applied to the local population (CASP10); and 11) the results of this study fit with other available evidence (CASP11).

\section{Statistical analysis}

The present meta-analysis was performed using Comprehensive Meta-analysis 2.0 software (Biostatic Inc., Englewood, NJ, USA). The strength of the association between the VEGF, eNOS, and $A B C B 1$ polymorphisms and ONFH risk was evaluated based on the odds ratio (OR) with corresponding $95 \% \mathrm{Cl}$, which were calculated using random effects or fixed effects models. The statistical significance of pooled the OR was assessed by a $Z$ test, and forest plots were drawn to reflect the $\mathrm{OR}$ with corresponding $95 \% \mathrm{Cl}$ in each group. Heterogeneity among studies was evaluated using the Cochran's $Q$-statistic; $P_{h}<0.05$ was considered to indicate heterogeneity. The extent of heterogeneity was analyzed applying $I^{2}$ test $(0 \%$, no heterogeneity; $100 \%$, maximum heterogeneity). If heterogeneity existed $\left(P_{h}<0.05\right.$ or $\left.I^{2}>50 \%\right)$, the random effects model was used to pool the data; otherwise, the fixed effects model was used. Sources of heterogeneity were explored by univariate and multivariate meta-regression analyses, and further verified using the Monte Carlo method. Each selected study was eliminated, and sensitivity analysis was used to assess the influence of a single study on the final results. Publication bias was estimated by applying funnel plots, classic fail-safe $\mathrm{N}$ test, and Egger's test to evaluate the reliability. All $\mathrm{P}$ values were 2-sided, and $\mathrm{P}<0.05$ was considered significant.

\section{RESULTS}

\section{Baseline characteristics}

There were 123 potential articles after duplicates $(N=12)$ were removed from the retrieved studies. Fifty-four of these studies were further excluded for various reasons after screening the abstracts (12 were letters, reviews and meta-analyses; 22 were non-human studies; 20 was not related to the research topic). Next, of the remaining 69 studies, a full review and detailed reading led to the exclusion of 57 studies (12 were not case-control studies; 20 were not relevant to ONHF; 25 were not relevant to VEGF, eNOS, and $A B C B 1$ ). As a final screening step, 2 of the 12 remaining studies were rejected because the data was irrelevant to the topic (Liu et al., 2012; Zheng et al., 2014), and thus 10 studies published between 2003 and 2013 (Asano et al., 2003; Koo et al., 2006; Yang and Xu, 2007; Kim et al., 2008; Kuribayashi et al., 2008; He and Li, 2009; Lee et al., 2012; Liu et al., 2012; Gagala et al., 2013; Kim et al., 2013) were analyzed in this meta-analysis. A total of 1025 patients with ONFH and 1730 healthy individuals were included, with sample sizes ranging from 48-807. Two of 10 included studies were performed in Caucasians, while 8 were in Asians. The single-nucleotide polymorphism (SNP) was identified using direct sequencing, polymerase chain reaction-restriction fragment length polymorphism, and TaqMan assays. The genotype distributions of included studies were in accordance with Hardy-Weinberg equilibrium (all P> 0.05). Baseline characteristics and CASP of included studies are as shown in Table 1 and Figure 1, respectively. 


\begin{tabular}{|c|c|c|c|c|c|c|c|c|}
\hline First author & Year & Country & Genotype method & Gene & SNP & Total & Case group & Control group \\
\hline Kim HS & 2013 & Korea & TaqMan & eNOS & rs2070744 T/C & 352 & 50 & 302 \\
\hline Gagala J & 2013 & Poland & PCR-RFLP & eNOS & rs2070744 T/C & 168 & 68 & 100 \\
\hline Liu B & 2012 & China & PCR-RFLP & VEGF & $-634 \mathrm{G} / \mathrm{C}$ & 440 & 220 & 220 \\
\hline Lee YJ & 2012 & Korea & PCR-RFLP & VEGF & $-634 \mathrm{G} / \mathrm{C}$ & 320 & 160 & 160 \\
\hline $\mathrm{He} W$ & 2009 & China & Direct sequencing & ABCB1 & $3435 \mathrm{C} / \mathrm{T}$ & 48 & 31 & 17 \\
\hline $\operatorname{Kim} \mathrm{T}$ & 2008 & Korea & TaqMan & VEGF & $-634 \mathrm{G} / \mathrm{C}$ & 807 & 312 & 495 \\
\hline Kuribayashi M & 2008 & Japan & Direct sequencing & ABCB1 & $3435 \mathrm{C} / \mathrm{T}$ & 151 & 30 & 121 \\
\hline Yang $\mathrm{X}$ & 2007 & China & PCR-RFLP & ABCB1 & $3435 \mathrm{C} / \mathrm{T}$ & 127 & 21 & 106 \\
\hline Koo KH & 2006 & USA & PCR-RFLP & eNOS & rs2070744 T/C & 206 & 103 & 103 \\
\hline Asano $\mathrm{T}$ & 2003 & Japan & PCR-RFLP & ABCB1 & $3435 \mathrm{C} / \mathrm{T}$ & 136 & 30 & 106 \\
\hline
\end{tabular}

PCR-RFLP = polymerase chain reaction with the restriction fragment length polymorphism; SNP = single-nucleotide polymorphism; TaqMan = TaqMan assay.

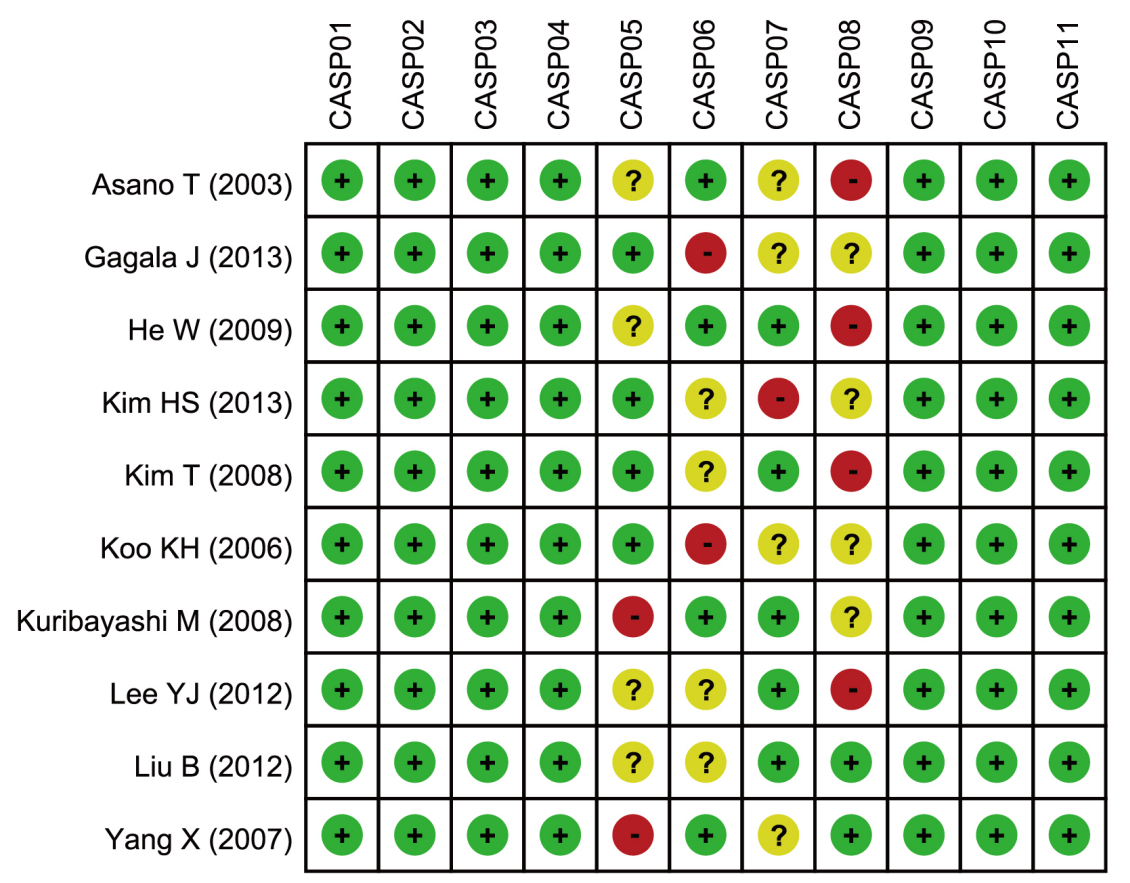

Figure 1. Assessment of the methodological quality of all 10 case-control studies was carried out using the CASP scores in our meta-analysis.

\section{Associations between the VEGF, eNOS, and $A B C B 1$ polymorphisms and ONFH}

Three of the 10 studies reported a correlation between the VEGF rs2010963 G>C polymorphism and the risk of ONFH. Heterogeneity was not observed among these studies, and thus the fixed effects model was used $(P>0.05)$. The results of our meta-analysis demonstrated that the VEGF rs2010963 G>C polymorphism was associated with an increased risk of ONFH (allele model: $\mathrm{OR}=1.252,95 \% \mathrm{Cl}=1.085-1.444, \mathrm{P}=0.002$; dominant model: $\mathrm{OR}=1.373,95 \% \mathrm{Cl}$ $=1.099-1.715, \mathrm{P}=0.005)$ (Figure 2 and Table 2$)$. 
A

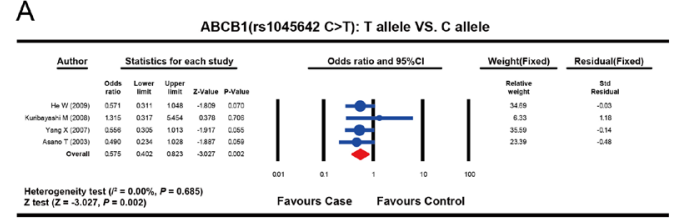

C

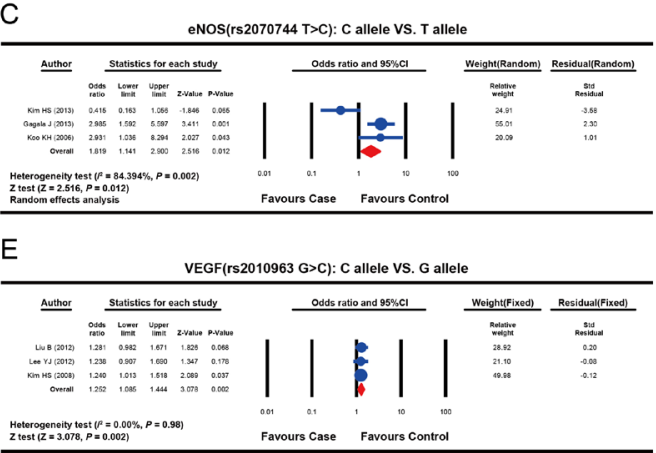

B

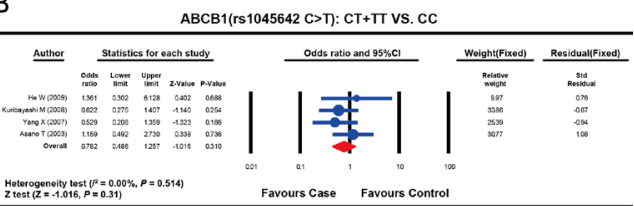

D

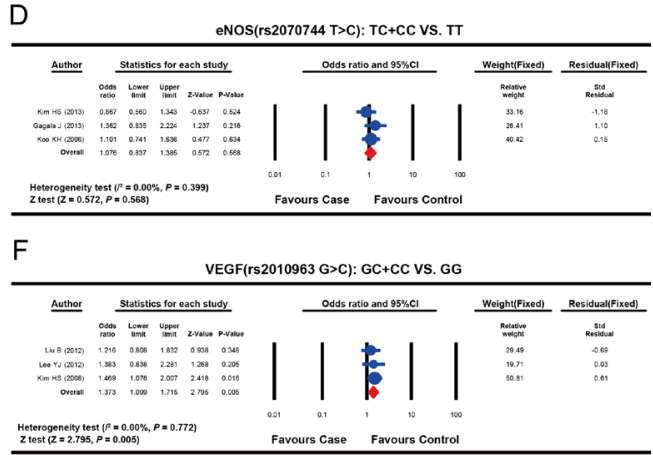

Figure 2. Forest plots for the correlations between the VEGF (rs2010963 G>C), eNOS (rs2070744 T>C), and ABCB1 (rs1045642 C>T) polymorphisms and ONFH risk.

Table 2. Comparisons of genotype and allele frequencies between the case and the control groups.

\begin{tabular}{|c|c|c|c|c|c|c|c|c|c|}
\hline \multirow[t]{2}{*}{ SNP } & \multicolumn{3}{|c|}{ rs 1045642 C > T } & \multicolumn{3}{|c|}{ rs2070744T/C } & \multicolumn{3}{|c|}{ rs2010963 G>C } \\
\hline & OR & $95 \% \mathrm{Cl}$ & $P$ & OR & $95 \% \mathrm{Cl}$ & $\mathrm{P}$ & OR & $95 \% \mathrm{Cl}$ & $\mathrm{P}$ \\
\hline Allele model & 0.575 & $0.40-0.82$ & 0.002 & 1.82 & $1.14-2.90$ & 0.012 & 1.25 & $1.09-1.44$ & 0.002 \\
\hline Dominant model & 0.78 & $0.49-1.26$ & 0.310 & 1.08 & $0.84-1.39$ & 0.568 & 1.37 & $1.10-1.71$ & 0.005 \\
\hline Homozygous model & 0.17 & $0.06-0.51$ & 0.047 & 4.04 & $0.62-26.15$ & 0.143 & 1.58 & $1.17-2.13$ & 0.03 \\
\hline Heterozygous model & 4.86 & $1.67-14.17$ & 0.004 & 0.41 & $0.06-2.76$ & 0.357 & 0.82 & $0.62-1.07$ & 0.148 \\
\hline Recessive model & 0.19 & $0.07-0.54$ & 0.002 & 0.29 & $0.05-1.86$ & 0.192 & 1.34 & $1.01-1.04$ & 0.026 \\
\hline
\end{tabular}

$\mathrm{SNP}=$ single nucleotide polymorphism; OR= odds ratio; $95 \% \mathrm{Cl}=95 \%$ confidence intervals.

Three of studies investigated the correlation between the eNOS rs2070744 T>C polymorphism and ONFH risk. Heterogeneity was found in the allele model among the included studies, and thus the random effects model was applied $(P=0.002)$. In contrast, no heterogeneity was detected in the dominant model; thus, the fixed effects model was utilized $(P=0.399)$. The results of our study showed that the eNOS rs2070744 T>C polymorphism was associated with an increased risk of ONFH under the allele model $(\mathrm{OR}=1.819,95 \% \mathrm{Cl}=1.141-2.900, \mathrm{P}=0.012)$, but no statistical significance was observed between the eNOS rs2070744 T>C polymorphism and ONFH under the dominant model $(\mathrm{OR}=1.076,95 \% \mathrm{Cl}=0.837-1.385, \mathrm{P}=0.568)$ (Figure 2 and Table 2).

Four of the 10 studies discussed the correlation between the $A B C B 1$ rs1045642 C>T polymorphism and ONFH risk. No heterogeneity was detected both in the allele model and the dominant model, and thus the fixed effects model was applied (all $P>0.05$ ). The meta-analysis results suggested that the $A B C B 1 \mathrm{rs} 1045642 \mathrm{C}>\mathrm{T}$ polymorphism significantly increased the risk of ONFH under the allele model $(\mathrm{OR}=0.575,95 \% \mathrm{Cl}=0.402-0.823, \mathrm{P}=0.002)$; however, no such association was detected between the $A B C B 1 \mathrm{rs} 1045642 \mathrm{C}>\mathrm{T}$ polymorphism and ONFH under the dominant model $(\mathrm{OR}=0.782,95 \% \mathrm{Cl}=0.486-1.257, \mathrm{P}=0.310)$ (Figure 2 and Table 2$)$. 


\section{Sensitivity analysis and publication bias}

The result of univariate meta-regression analyses illustrated that publication year, ethnicity, sample size, SNP, and genotyping method were not sources of heterogeneity and key factors of overall effect size (all $P>0.05$ ), which was further confirmed using multivariate meta-regression analysis (Figure 3 and Table 3). Sensitivity analysis results suggested that each included study had no impact on the pooled OR of the VEGF rs2010963 G>C, eNOS rs2070744 T>C, and ABCB1 rs1045642 C>T SNPs and the risk ONFH (Figure 4). The shape of the funnel plots showed no obvious asymmetry in differences of allele gene and genotype frequencies of rs2010963 G>C, rs2070744 T>C, and rs1045642 C>T among case and control groups, suggesting no evidence of publication bias. Classic fail-safe $\mathrm{N}$ and Egger's test further confirmed that no publication bias existed $(P>0.05)$ (Figure 5).

A

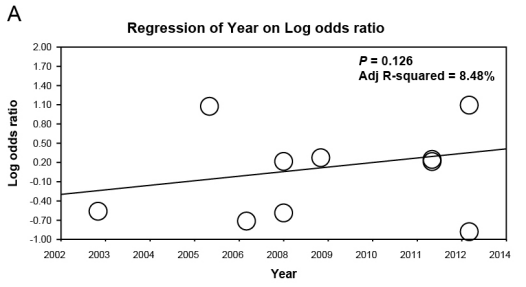

C

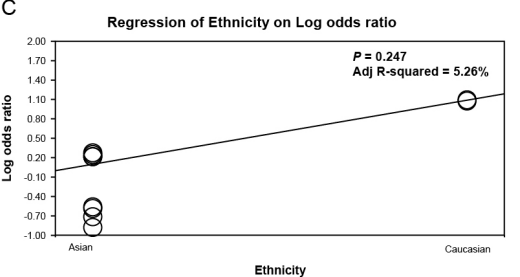

E

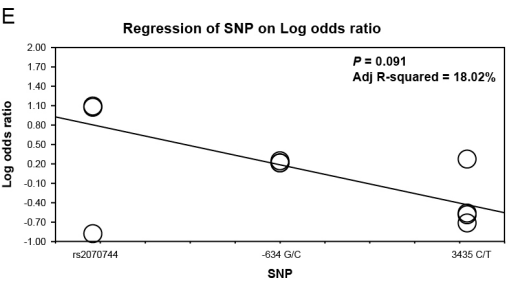

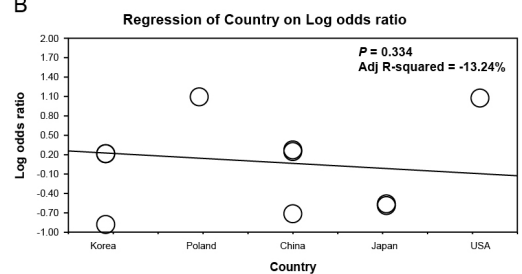

D

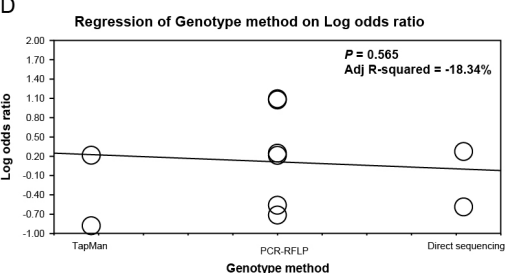

$\mathrm{F}$

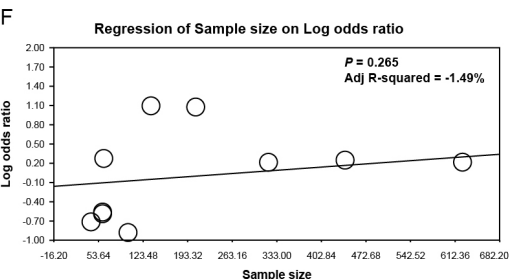

Figure 3. Meta-regression analysis examining the correlations between theVEGF (rs2010963 G>C), eNOS (rs2070744 $\mathrm{T}>\mathrm{C}$ ), and $A B C B 1$ (rs1045642 $\mathrm{C}>\mathrm{T}$ ) polymorphisms and ONFH risk.

\begin{tabular}{|c|c|c|c|c|c|c|}
\hline \multirow[t]{2}{*}{ Heterogeneity factors } & \multirow[t]{2}{*}{ Coefficient } & \multirow[t]{2}{*}{ SE } & \multirow[t]{2}{*}{$\mathrm{t}$} & \multirow[t]{2}{*}{$P$ (Adjusted) } & \multicolumn{2}{|c|}{$95 \% \mathrm{Cl}$} \\
\hline & & & & & $\mathrm{LL}$ & UL \\
\hline Year & -0.002 & 0.035 & -0.07 & 1.000 & -0.115 & 0.110 \\
\hline Country & 0.045 & 0.072 & 0.63 & 0.916 & -0.185 & 0.275 \\
\hline Ethnicity & -0.130 & 0.235 & -0.55 & 0.935 & -0.879 & 0.619 \\
\hline Method & -0.220 & 0.204 & -1.08 & 0.703 & -0.870 & 0.430 \\
\hline SNP & 0.096 & 0.192 & 0.50 & 0.953 & -0.517 & 0.710 \\
\hline Sample & -0.00 & 0.00 & -1.95 & 0.339 & -0.001 & 0.00 \\
\hline
\end{tabular}

SE = standard error; LL = lower limit; UL = upper limit. 
A
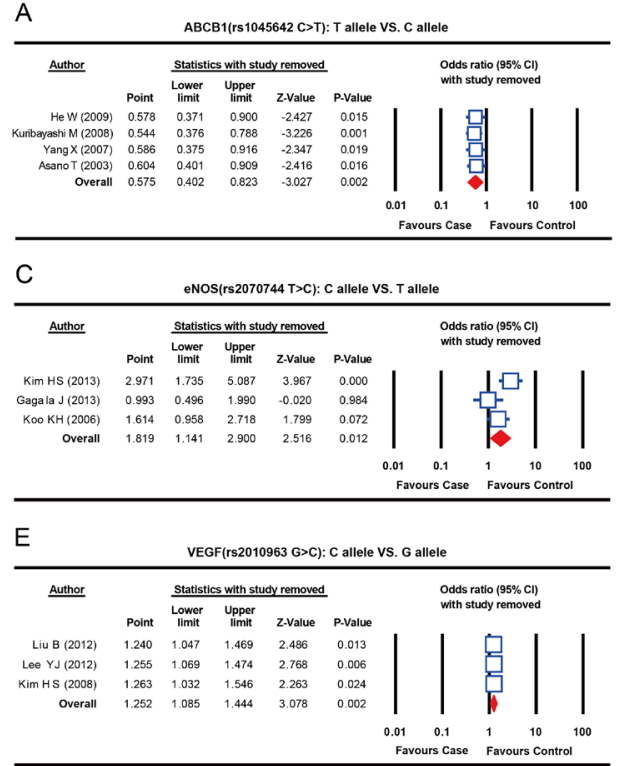

B

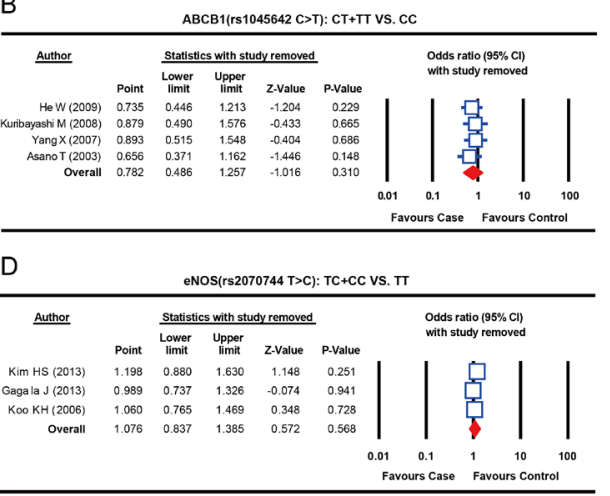

$\mathrm{F}$

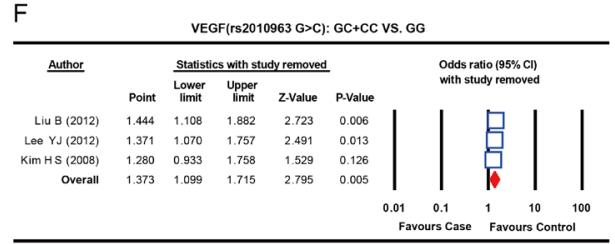

Figure 4. Sensitivity analysis examiningthe correlations between the VEGF (rs2010963 G>C), eNOS (rs2070744 $\mathrm{T}>\mathrm{C}$ ), and $A B C B 1$ (rs1045642 C>T) polymorphisms and ONFH risks.

A ABCB1(rs1045642 CrT): Tallele vs. Callele

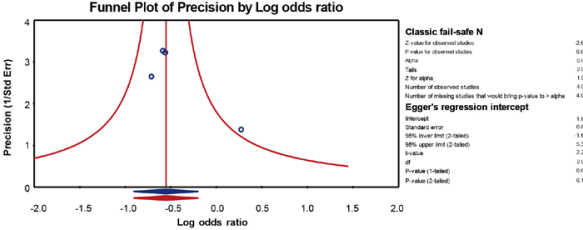

C

eNOS(rs2070744 I>C): C allele VS. T allele
Funnel Plot of Precision by Log odds ratio

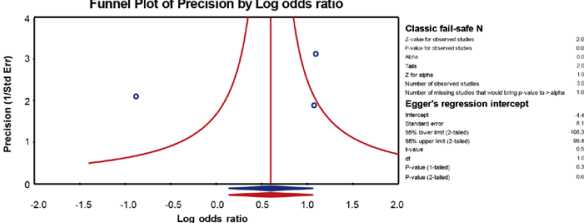

E
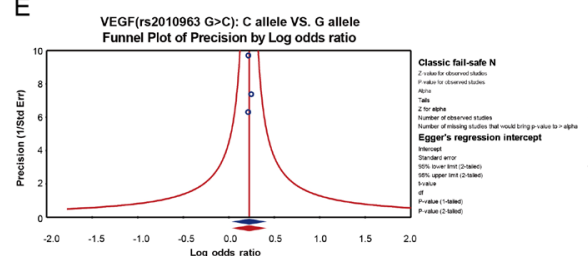

B

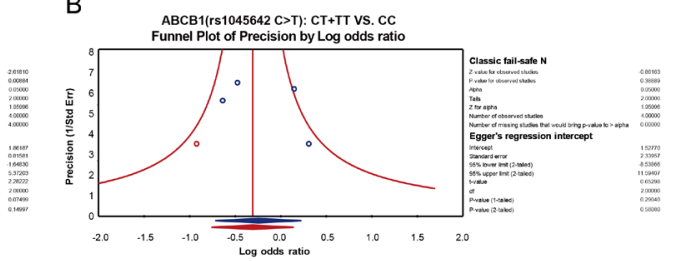

D

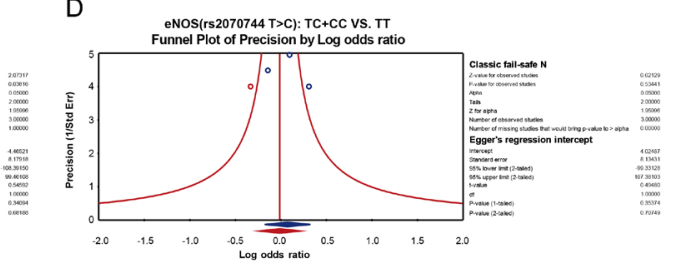

$\mathrm{F}$

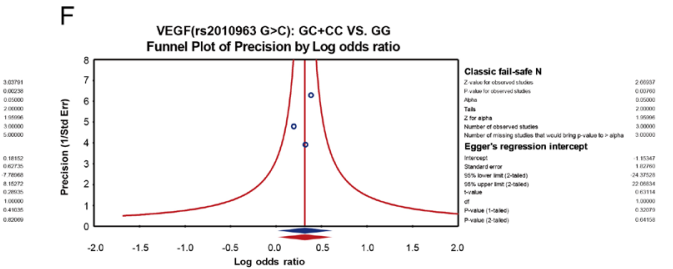

Figure 5. Publication bias for the correlations between the VEGF (rs2010963 G>C), eNOS (rs2070744 T>C), and $A B C B 1$ (rs1045642 C>T) polymorphisms and ONFH risks. 


\section{DISCUSSION}

The results of our meta-analysis support the hypothesis that SNPs in VEGF, eNOS, and $A B C B 1$ are independent risk factors for the development of ONFH. Destruction of the bone marrow and osteocyte integrity, which occurs because of wides pread cell death caused by inadequate blood supply, influences the segment of subchondral bone and is a typical feature observed in ONFH (Malizos et al., 2007). Multiple risk factors such as inflammatory disease, glucocorticoids, alcohol consumption, and liquid nitrogen, promote non-traumatic ONFH (Gao et al., 2013). Notably, impaired angiogenesis may be aterminal step in the pathways leading to the origin and progression of ONFH (Gao et al., 2013). VEGF is an important molecule in angiogenesis and plays a vital role in bone formation. For instance, normal growth plate morphogenesis, including blood vessel invasion and cartilage remodeling, is mediated by VEGF; VEGF functions also are critical in bone repair (Wernike et al., 2010; Jabbarzadeh et al., 2012). The VEGF polymorphisms located in the 5'-promoter region are important for the strength of VEGF gene expression (Liu et al., 2012). Previous studies reported that the VEGF 634G >C (rs2010963) polymorphism resulted in differential expression of VEGF, with the highest expression observed in GG homozygotes and the lowest observed in CC homozygotes (Vailati et al., 2012; Oh et al., 2013; Qiu et al., 2013). It has been suggested that VEGF levels in the bone necrosis area affect angiogenesis and repair processes, as well as influence the progression and outcome of ONFH, which agrees with our results that the VEGF rs2010963 G>C polymorphism increased the risk of ONFH (Kim et al., 2008).

Previous studies have suggested that eNOS, the main NOS isoform expressed in normal bone, modulates bone resorption and osteoclast formation. Additionally, the anabolic effects of estrogen and insulin-like growth factor depend, in part, on the NO produced by eNOS (Lagumdzija et al., 2004; Ponnuswamy et al., 2012; Kim et al., 2013). Sharma et al. suggested that subjects carrying the TT genotype exhibit decreased NO production compared with the GG and GT genotypes, indicating that the activity of eNOS is correlated with the presence of the $G$ allele (Sharma et al., 2014). Polymorphisms in eNOS that cause lower NO synthesis because of reduced NOS expression have been identified, and some of the polymorphisms correlate with the risk of ONFH (Paleolog et al., 1991; Kim et al., 2013). In the present study, our results demonstrated that the eNOS rs2070744 T>C polymorphism increased the risk of ONFH under the allele model. Our results also showed that the $A B C B 1 \mathrm{rs} 1045642 \mathrm{C}>\mathrm{T}$ polymorphism, under the allele model, increased the risk of ONFH. $A B C B 1$ encodes $\mathrm{P}$-gp, which is involved in the active transport of corticosteroids and is linked to the pathology of ONFH, potentially involving steroid metabolism (Zhang et al., 2014). The ABCB1 rs1045642 C>T polymorphism was found to be correlated with altered P-gp expression as well as abnormal P-gp function, and ONFH patients with the 3435TT genotype have high plasma digoxin levels compared with patients with the 3435CC genotype (Yang and $\mathrm{Xu}, 2007$ ).

There were some limitations to this meta-analysis. First, we focused only on studies published in the Chinese and English, representing a potential source of bias. Second, only 2 studies examined Caucasian populations, while 8 studies were reported in Asians. Finally, the sample size in our study was small, which may have influenced the results.

In conclusion, the VEGF rs2010963 G>C, eNOS rs2070744 T>C, and ABCB1 rs 1045642 $\mathrm{C}>\mathrm{T}$ polymorphisms significantly increased the risk of ONFH based on our meta-analysis. However, further studies including larger sample sizes are needed to confirm our findings. 


\section{Conflicts of interest}

The authors declare that they have no conflict of interest.

\section{ACKNOWLEDGMENTS}

We would like to acknowledge the reviewers for their helpful comments on this paper.

\section{REFERENCES}

Alzoubi KH, Khabour OF, Al-Azzam SI and Mayyas F (2013). The role of multidrug resistance-1 (MDR1) variants in response to fexofenadine among Jordanians. Int. J. Clin. Pharmacol. Ther. 51: 880-887.

Asano T, Takahashi KA, Fujioka M, Inoue S, et al. (2003). ABCB1 C3435T and G2677T/A polymorphism decreased the risk for steroid-induced osteonecrosis of the femoral head after kidney transplantation. Pharmacogenetics 13: 675-682.

Cariboni A, Davidson K, Dozio E, Memi F, et al. (2011). VEGF signalling controls GnRH neuron survival via NRP1 independently of KDR and blood vessels. Development 138: 3723-3733.

Dangel O, Mergia E, Karlisch K, Groneberg D, et al. (2010). Nitric oxide-sensitive guanylyl cyclase is the only nitric oxide receptor mediating platelet inhibition. J. Thromb. Haemost. 8: 1343-1352.

Foxton RH, Finkelstein A, Vijay S, Dahlmann-Noor A, et al. (2013). VEGF-A is necessary and sufficient for retinal neuroprotection in models of experimental glaucoma. Am. J. Pathol. 182: 1379-1390.

Fung KL and Gottesman MM (2009). A synonymous polymorphism in a common MDR1 (ABCB1) haplotype shapes protein function. Biochim. Biophys. Acta 1794: 860-871.

Gagala J, Buraczynska M, Mazurkiewicz T and Ksiazek A (2013). Endothelial nitric oxide synthase gene intron 4 polymorphism in non-traumatic osteonecrosis of the femoral head. Int. Orthop. 37: 1381-1385.

Gao YS, Wang HF, Ding H and Zhang CQ (2013). A novel rat model of osteonecrosis of the femoral head induced by periarticular injection of vascular endothelial growth factor receptor 2 antibody. J. Surg. Res. 183: e1-e5.

García M, Macías RM, Cubero JJ, Benítez J, et al. (2013). ABCB1 polymorphisms are associated with cyclosporine-induced nephrotoxicity and gingival hyperplasia in renal transplant recipients. Eur. J. Clin. Pharmacol. 69: 385-393.

Guan X, Zhao H, Niu J, Tang D, et al. (2009). The VEGF -634G>C promoter polymorphism is associated with risk of gastric cancer. BMC Gastroenterol. 9: 77.

Han N, Yan ZQ, Guo CA, Shen F, et al. (2010). Effect of rifampicin on the risk of steroid-induced osteonecrosis of the femoral head. Orthop. Surg. 2: 124-133.

Harvey NC, Lillycrop KA, Garratt E, Sheppard A, et al. (2012). Evaluation of methylation status of the eNOS promoter at birth in relation to childhood bone mineral content. Calcif. Tissue Int. 90: 120-127.

He W and Li K (2009). Incidence of genetic polymorphisms involved in lipid metabolism among Chinese patients with osteonecrosis of the femoral head. Acta Orthop. 80: 325-329.

Herrera BS, Martins-Porto R, Maia-Dantas A, Campi P, et al. (2011). iNOS-derived nitric oxide stimulates osteoclast activity and alveolar bone loss in ligature-induced periodontitis in rats. J. Periodontol. 82: 1608-1615.

Higashibata T, Hamajima N, Naito M, Kawai S, et al. (2012). eNOS genotype modifies the effect of leisure-time physical activity on serum triglyceride levels in a Japanese population. Lipids Health Dis. 11: 150.

Jabbarzadeh E, Deng M, Lv Q, Jiang T, et al. (2012). VEGF-incorporated biomimetic poly (lactide-co-glycolide) sintered microsphere scaffolds for bone tissue engineering. J. Biomed. Mater. Res. B Appl. Biomater. 100: 2187-2196.

Kim HS, Bae SC, Kim TH andKim SY (2013). Endothelial nitric oxide synthase gene polymorphisms and the risk of osteonecrosis of the femoral head in systemic lupus erythematosus. Int. Orthop. 37: 2289-2296.

Kim T, Hong JM, Lee J, Oh B, et al. (2008). Promoter polymorphisms of the vascular endothelial growth factor gene is associated with an osteonecrosis of the femoral head in the Korean population. Osteoarthritis Cartilage 16: 287-291.

Kim TH, Hong JM, Shin ES, Kim HJ, et al. (2009). Polymorphisms in the annexin gene family and the risk of osteonecrosis of the femoral head in the Korean population. Bone 45: 125-131.

Koo KH, Lee JS, Lee YJ, Kim KJ, et al. (2006). Endothelial nitric oxide synthase gene polymorphisms in patients with nontraumatic femoral head osteonecrosis. J. Orthop. Res. 24: 1722-1728.

Lagumdzija A, Ou G, Petersson M, Bucht E, et al. (2004). Inhibited anabolic effect of insulin-like growth factor-I on stromal bone marrow cells in endothelial nitric oxide synthase-knockout mice. Acta Physiol. Scand. 182: 29-35.

Lee YJ, Lee JS, Kang EH, Lee YK, et al. (2012). Vascular endothelial growth factor polymorphisms in patients with steroid- 
induced femoral head osteonecrosis. J. Orthop. Res. 30: 21-27.

Li M, Tan J, Yang X, Su L, et al. (2014). The ABCB1-C3435T polymorphism likely acts as a risk factor for resistance to antiepileptic drugs. Epilepsy Res. 108: 1052-1067.

Liang XN, Xie L, Cheng JW, Tan Z, et al. (2013). Association between PAI-1 4G/5G Polymorphisms and osteonecrosis of femoral head: a meta-analysis. Thromb. Res. 132: 158-163.

Liu B, Cao Y, Wang D, Yao G, et al. (2012). Vascular endothelial growth factor $-634 \mathrm{G} / \mathrm{C}$ polymorphism associated with osteonecrosis of the femoral head in a Chinese population. Genet. Test. Mol. Biomarkers 16: 739-743.

Liu CH, Zhao DW and Wang BJ (2012). [Effects of different stress force stimulations on the expression of vascular endothelial growth factor in Beagle dogs in the repairing process of osteonecrosis of the femoral head]. Zhonghua Yi Xue Za Zhi. 92: 40-44.

Malizos KN, Karantanas AH, Varitimidis SE, Dailiana ZH, et al. (2007). Osteonecrosis of the femoral head: etiology, imaging and treatment. Eur. J. Radiol. 63: 16-28.

Mehrab-Mohseni M, Tabatabaei-Malazy O, Hasani-Ranjbar S, Amiri P, et al. (2011). Endothelial nitric oxide synthase VNTR (intron $4 \mathrm{a} / \mathrm{b}$ ) polymorphism association with type 2 diabetes and its chronic complications. Diabetes Res. Clin. Pract. 91: 348-352.

Oh SY, Kwon HC, Kim SH, Lee S, et al. (2013). The relationship of vascular endothelial growth factor gene polymorphisms and clinical outcome in advanced gastric cancer patients treated with FOLFOX: VEGF polymorphism in gastric cancer. BMC Cancer 13: 43.

Paleolog EM, Carew MA and Pearson JD (1991). Effects of tumour necrosis factor and interleukin-1 on von Willebrand factor secretion from human vascular endothelial cells. Int. J. Radiat. Biol. 60: 279-285.

Ponnuswamy P, Schröttle A, Ostermeier E, Grüner S, et al. (2012). eNOS protects from atherosclerosis despite relevant superoxide production by the enzyme in apoE mice. PLoS One 7: e30193.

Qiu M, Xiong W, Liao H and Li F (2013). VEGF -634G>C polymorphism and diabetic retinopathy risk: a meta-analysis. Gene 518: 310-315.

Sharma D, Hussain SA, Akhter N, Singh A, et al. (2012). Endothelial nitric oxide synthase (eNOS) gene Glu298Asp polymorphism and expression in North Indian preeclamptic women. Pregnancy Hypertens. 4: 65-69.

Vailati FB, Crispim D, Sortica DA, Souza BM, et al. (2012). The C allele of -634G/C polymorphism in the VEGFA gene is associated with increased VEGFA gene expression in human retinal tissue. Invest. Ophthalmol. Vis. Sci. 53: 6411-6415.

Wang W, Li S, Niu D, Liu F, et al. (2011). Relationship between the bone mass and the expressions of vascular endothelial growth factor, basic fibroblast growth factor, and bone morphogenetic protein 2 mRNA in avascular necrosis of femoral head. Zhongguo Xiu Fu Chong Jian Wai Ke Za Zhi 25: 984-991.

Wernike E, Montjovent MO, Liu Y, Wismeijer D, et al. (2010). VEGF incorporated into calcium phosphate ceramics promotes vascularisation and bone formation in vivo. Eur. Cell Mater. 19: 30-40.

Yamaguchi R, Yamamoto T, Motomura G, Ikemura S, et al. (2011). Incidence of nontraumatic osteonecrosis of the femoral head in the Japanese population. Arthritis Rheum. 63: 3169-3173.

Yang XY andXu DH (2007). MDR1 (ABCB1) gene polymorphisms associated with steroid-induced osteonecrosis of femoral head in systemic lupus erythematosus. Pharmazie 62: 930-932.

Zhang Y, Kong X, Wang R, Li S, et al. (2014). Genetic association of the P-glycoprotein gene ABCB1 polymorphisms with the risk for steroid-induced osteonecrosis of the femoral head in Chinese population. Mol. Biol. Rep. 41: 3135-3146.

Zheng L, Wang W, Ni J, Li Z, et al. (2014). The association of eNOS gene polymorphism with avascular necrosis of femoral head. PLoS One 9: e87583. 